\title{
MICROCIRCULATION OF THE URINARY BLADDER IN A RAT MODEL OF ISCHEMIA-REPERFUSION-INDUCED CYSTITIS
}

\author{
ZOLTÁN BAJORY, JÖRG HUTTER, FRITZ KROMBACH, AND KONRAD MESSMER
}

\begin{abstract}
Objectives. To determine the microcirculatory disturbances in a rat model of ischemia-reperfusion-induced cystitis using intravital fluorescence videomicroscopy.

Methods. Twenty male Sprague-Dawley rats were used for the experiments. In 10 animals, warm ischemia of the bladder was induced for 60 minutes. After 30 minutes of reperfusion, microvascular macromolecular leakage, leukocyte-endothelial cell interactions, venular red blood cell velocity, functional capillary density, and the arteriolar and venular diameters were determined by intravital videomicroscopy. In addition, the intravesical pressure and macrohemodynamic parameters were assessed during the experiments. Shamoperated animals served as the controls $(n=10)$.

Results. After ischemia-reperfusion, the numbers of rolling and firmly adherent leukocytes in the postcapillary venules were significantly increased. Venular red blood cell velocity and functional capillary density, as well as the arteriolar and venular diameters, were significantly decreased. The macromolecular leakage had increased in both arterioles and venules.

Conclusions. After ischemia-reperfusion, inflammatory reactions and microcirculatory failure were observed in the urinary bladder. This study targeted the microcirculatory consequences of cystitis using intravital videomicroscopy. Because the parameters investigated are relevant not only for ischemia-reperfusion of the urinary bladder but also for cystitis caused by other stimuli, this model represents a novel tool in the field of inflammation research in urology. UROLOGY 60: 1136-1 140, 2002. () 2002, Elsevier Science Inc.
\end{abstract}

$\mathbf{I}^{\mathrm{n}}$ nflammation of the urinary bladder is a frequent diagnosis in clinical practice and is widely investigated in clinical, as well as experimental, research, but many aspects of the pathogenesis are still not completely understood. Several experimental animal models have been established inducing inflammation of the urinary bladder in various ways to mimic the pathogenesis occurring in humans. ${ }^{1-4}$ Ischemia-reperfusion (I/R) is widely used in experimental research as a trigger of inflammation in various organs. ${ }^{5,6}$ To date, it has not

This study was supported by the European Urological Scholarship Program (EUSP) of the European Association of Urology (EAU) and OTKA: F 032069.

From the Institute for Surgical Research, University of Munich, Klinikum Grosshadern, Munich, Germany; and Department of Urology and Institute of Surgical Research, University of Szeged, Albert Szent-Györgyi Medical Center, Szeged, Hungary.

Reprint requests: Zoltán Bajory, M.D., Department of Urology, University of Szeged, Kalvaria sgt. 57, Szeged 6725, Hungary

Submitted: January 29, 2002, accepted (with revisions): June 21,2002 been used to induce cystitis, even though I/R also can be an etiologic factor for cystitis in patients.

$\mathrm{I} / \mathrm{R}$ of the urinary bladder can result from various pathologic conditions, including bladder overdistension caused by lower urinary tract obstruction, thromboembolization and recanalization, infrarenal aortic aneurysm, temporary ligation of cystic arteries, complications of pregnancy, or from hemorrhagic or septic shock. Although re-establishment of perfusion is mandatory for the survival of ischemic organs, reperfusion is associated with local postischemic inflammation and thereby paradoxically promotes additional tissue injury. ${ }^{5-8}$ In the bladder, the consequences of $\mathrm{I} / \mathrm{R}$ injury can range from increased urothelial permeability to necrosis in the worst case.

In general, impairment of nutritive organ perfusion and the adherence of activated leukocytes to the endothelial surface are the key events in the development of acute inflammatory responses. Although it is known from studies in various organs that a complex sequence of chemical and cellular 
reactions is initiated during $\mathrm{I} / \mathrm{R}$, leading to alterations of microvascular flow and leukocyte-endothelial cell interaction, our study established for the first time the feasibility of intravital fluorescence video microscopic (IVM) analysis of these microcirculatory phenomena in the urinary bladder. We developed a standardized rat model to study the microcirculation of the urinary bladder in a reproducible manner under normal and pathologic conditions. In the present study, we describe the impact of $\mathrm{I} / \mathrm{R}$ on the microcirculation of the urinary bladder and characterize $\mathrm{I} / \mathrm{R}$ as a trigger for cystitis.

\section{MATERIAL AND METHODS}

\section{Animals}

Twenty male Sprague-Dawley rats (average weight $270 \mathrm{~g}$ ) were housed in an environmentally controlled room with a 12-hour light-dark cycle and free access to food and water. The animals were randomly assigned to two groups $(n=10)$. The experiments were performed in accordance with the German legislation on animal protection.

\section{Surgical Procedure and Experimental Protocol}

After administration of atropine sulfate $(0.1 \mathrm{mg} / \mathrm{kg}$ body weight; Braun, Melsungen, Germany) subcutaneously as premedication and sodium pentobarbital $(45 \mathrm{mg} / \mathrm{kg}$ body weight; Narcoren, Merial GmbH, Hallbergmoos, Germany) intraperitoneally as anesthesia, the animals were placed in the supine position on a heating pad to maintain normal body temperature during the experiments. All surgical procedures were made with the help of an operation microscope (M651, Leica, Bensheim, Germany). Polyethylene catheters (internal diameter $0.28 \mathrm{~mm}$, outer diameter $0.61 \mathrm{~mm}$; SIMS Portex, Hythe, UK) were inserted into the carotid artery and jugular vein to measure the mean arterial pressure and heart rate (Plugsys, Hugo Sachs Elektronik, March, Germany) and for the injection of fluorescent dyes. The animals were tracheotomized and cannulated (Abbocath-T, 13G, Abbott, Sligo, Ireland).

After a midline laparotomy, the bladder was exteriorized, and the median umbilical ligament was cut. The urethra was ligated with 4-0 Perma-Hand silk suture (Johnson \& Johnson, Brussels, Belgium). The ureters were dissected at their middle part to avoid bladder distension due to urinary inflow. A polyethylene catheter (internal diameter $0.28 \mathrm{~mm}$, outer diameter $0.61 \mathrm{~mm}$, SIMS Portex) was inserted into the bladder at the dome. The bladder was filled with $0.5 \mathrm{~mL}$ of a body warm physiologic sodium chloride solution. The intravesical pressure was continuously monitored (Plugsys).

In the one group, warm ischemia of the urinary bladder was induced by clamping the cystic artery branches using two small metal clips (I/R group). Afterward, the abdominal wall was closed by clips. After 60 minutes, the abdomen was reopened, and the clips were removed, allowing for a 30-minute period of reperfusion before IVM observation was performed. For this purpose, the bladder was placed on an adjustable stage by gently pulling at the stump of the umbilical ligament. In the control group, the IVM measurements were performed 90 minutes after a sham operation. In a couple of experiments, the bladder was resected, fixed in formalin (4\%), embedded in paraffin, sectioned, and stained with hematoxylin-eosin for histologic examination. Finally, the animals were killed with an overdose of sodium pentobarbital.

\section{INTRAVITAL VIDEOMICROSCOPY}

Plasma staining was achieved by intravenous injection of fluorescein isothiocyanate-labeled albumin $(0.2 \mathrm{~mL}, 4 \%$, molecular weight 70000, Sigma Chemical, St. Louis, Mo), and rhodamine $6 \mathrm{G}(0.1 \mathrm{~mL}, 0.2 \%$, molecular weight 479 , Sigma) was used for the labeling of the leukocytes. The microcirculation of the bladder was visualized using a high-resolution, modified Leitz-Orthoplan microscope attached to a PloemoPak illuminator with an I2/3 blue (excitation filter $495 \mathrm{~nm}$, emission filter $515 \mathrm{~nm}$ ) and an N2 green (exitation filter 525 $\mathrm{nm}$, emission filter $555 \mathrm{~nm}$ ) filter block (Leitz, Wetzlar, Germany). The microcirculatory network was analyzed with the epi-illumination technique (HBO, 100W lamp, Osram, Munich, Germany). With a $25 \times$ water immersion objective (W $25 \times / 0.6$, Leitz), the magnification was $540 \times$ on the video screen (Sony, Tokyo, Japan). The microscopic images were recorded by a charge-coupled device video camera (FK 6990, Pieper GmbH, Schwerte, Germany) attached to an S-VHS video recorder (BR-S920E, JVC, Tokyo, Japan).9,10

\section{VIDEO ANALYSIS}

Quantitative analysis of the microcirculatory parameters was performed off-line using a computer-assisted analysis system (CAMAS, Dr. H. Zeintl, Heidelberg, Germany). ${ }^{11,12}$ The venular and arteriolar diameters, venular red blood cell velocity, functional capillary density (FCD) or length of erythrocyte-perfused capillaries per observation area, and macromolecular leakage (extravascular/intravascular fluorescence intensity ratio) were determined within five observation fields. ${ }^{10}$ The numbers of rolling and adherent leukocytes were evaluated as a measure of the leukocyte-endothelial cell interaction $^{12}$ in five venular segments per animal. Rolling leukocytes were defined as cells moving significantly slower than red blood cells in the centerline of the vessel and are given as the number of rolling leukocytes per vessel diameter per second. Firmly adherent leukocytes were identified in each vessel segments as cells that did not detach from the endothelial lining within 30 seconds and are given as the number of cells per square millimeter of endothelial surface, calculated from the diameter and length of the venular segment, assuming cylindric geometry. ${ }^{10-12}$

\section{Statistical AnALYSIS}

The statistical analysis was performed with a statistical software package (SigmaStat 2.0 for Windows, Jandel Scientific, Germany). The Mann-Whitney rank sum test was applied for comparisons between the two groups. The mean \pm standard deviation is given. $P<0.05$ was considered significant.

\section{RESULTS}

After filling the bladder with $0.5 \mathrm{~mL}$ saline solution, the intravesical pressure was $9 \pm 2 \mathrm{~mm} \mathrm{Hg}$ in both groups and did not change significantly during the experiments. The macrohemodynamic parameters were in the physiologic range and did not differ between the two groups (data not shown).

At the microcirculatory level of the bladder wall, all investigated parameters were significantly changed after I/R compared with the control group. The numbers of rolling and firmly adherent leukocytes in postcapillary venules were elevated by $527 \%$ and $4637 \%$, respectively (Fig. 1). The macromolecular leakage in the arterioles and venules was also significantly increased by $33 \%$. 


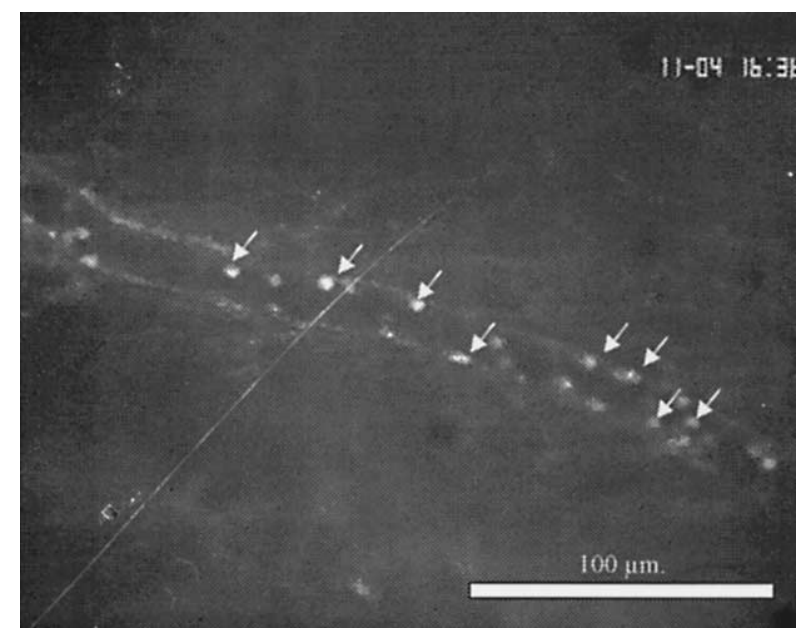

FIGURE 1. Postcapillary venule in the muscular layer of the urinary bladder. Rhodamine-6G-labeled leukocytes rolling on and sticking (arrows) to the endothelium during reperfusion.

The venular red blood cell velocity and FCD decreased to $36 \%$ and $28 \%$ of the control values, respectively. The arteriolar and venular diameters were also reduced to $73 \%$ of the controls (Table I).

The histologic examination of the hematoxylineosin-stained sections revealed increased lymphocyte and polymorphonuclear leukocyte migration into the submucosa, lamina propria, and epithelium. Diffuse edema was observed in all layers of the bladder wall.

\section{COMMENT}

The inflammatory mechanisms underlying the different types of cystitis have been investigated using various animal models. After administration of cyclophosphamide, the development of cystitis was reported in rats. ${ }^{1,13}$ In this type of cystitis, plasma protein extravasation was significantly increased. ${ }^{1}$ Cystitis also can be induced by intravesical instillation of formalin, capsaicin, or lipopolysaccharides. A foreign body in the bladder can cause cystitis by mechanical stimulation, infection, or immunologic reaction. Interstitial cystitis was induced by administration of bladder homogenate in complete Freund's adjuvant on the basis of a hypothesis of autoimmune pathogenesis. ${ }^{14}$ Intramural edema, recruitment of inflammatory cells, and mastocytosis were found in all forms of inflammation. ${ }^{3}$ To date, I/R has not been reported as a model for cystitis, even though this pathologic mechanism is widely accepted as a trigger of inflammation in other organs. ${ }^{5,6}$

Hypoxia and reoxygenation of a tissue initiates a sequence of inflammatory reactions, during the course of which no single process can be identified as the critical event. ${ }^{15}$ The main target of the $\mathrm{I} / \mathrm{R}$ injury is the microcirculation. I/R-induced microvascular failure is characterized by decreased capillary perfusion, termed "no-reflow," and quantifiable as decreased FCD, as well as by reflowassociated events, termed "reflow paradox," including enhanced leukocyte-endothelial cell interaction and increased microvascular permeability. ${ }^{16}$ The loss of FCD is mainly due to endothelial cell swelling, with subsequent entrapment of blood cells in capillaries, and prolongs local tissue hypoxia at the time of reperfusion, resulting in impaired energy metabolism and cell death. Inflammatory reactions such as leukocyte activation and adhesion during reperfusion can also contribute to organ injury. ${ }^{5-8}$

The body of evidence indicating that reactive oxygen species play an important role in mediating the vascular and parenchymatous injury is large. ${ }^{17}$ The oxygen radical hypothesis of reperfusion injury is based on the assumption that an additional tissue injury occurs during reperfusion and is dependent on the reintroduction of oxygen followed by the production of reactive oxygen species. A potential source of reactive oxygen species and mediator of injury in postischemic tissues is the polymorphonuclear leukocyte. Neutrophils contain nicotinamide-adenine dinucleotide phosphate (NADPH) oxidase that reduces molecular oxygen to superoxide anion. Activated neutrophils secrete the enzyme myeloperoxidase, which catalyzes the formation of hydrochlorous acid from hydrogen peroxide and chloride ions. Activated neutrophils can also release a variety of proteolytic enzymes that can injure the microvasculature. Thus, the neutrophil has the potential to be a source for the effects of $\mathrm{I} / \mathrm{R}$.

Increased neutrophil adhesiveness to the microvascular endothelium during reperfusion appears to be a critical first step in the pathogenesis of I/Rinduced injury to the microvasculature. A number of factors influence the adhesive interactions between neutrophils and endothelial cells. Xanthine oxidase-derived oxidants mediate I/R-induced neutrophil infiltration by enhancing the adhesive interactions between circulating neutrophils and the endothelial cells of postcapillary venules. Whether leukocytes adhere to venular endothelium also largely depends on the expression of adhesion molecules on the surface of activated neutrophils and/or endothelial cells, hydrodynamic dispersal forces (eg, blood flow velocity) that tend to sweep leukocytes away from the vascular wall, and products of neutrophil activation (eg, elastase). IVM has been used to monitor leukocyteendothelium interactions and other microcirculatory parameters, such as microvascular perfusion, macromolecular extravasation from microvessels, and changes in the caliber of arterioles and 
TABLE I. Microcirculatory changes during I/R-induced cystitis in rats

\begin{tabular}{|c|c|c|}
\hline & $\begin{array}{c}\text { Control Group } \\
(n=10)\end{array}$ & $\begin{array}{l}\text { I/R Group } \\
(n=10)\end{array}$ \\
\hline Venular diameter $(\mathrm{n}=5)(\mu \mathrm{m})$ & $40.7 \pm 6.4$ & $29.2 \pm 4.6$ \\
\hline Arteriolar diameter $(\mathrm{n}=5)(\mu \mathrm{m})$ & $39.7 \pm 7.1$ & $29.9 \pm 4.8$ \\
\hline $\operatorname{RBCV}(\mathrm{n}=5)(\mathrm{mm} / \mathrm{s})$ & $1.16 \pm 0.2$ & $0.42 \pm 0.1$ \\
\hline $\mathrm{FCD}(\mathrm{n}=5)(1 / \mathrm{cm})$ & $145 \pm 15.6$ & $41.2 \pm 6.3$ \\
\hline Venular macromolecular leakage $(n=5)$ & $0.71 \pm 0.1$ & $0.96 \pm 0.2$ \\
\hline Arteriolar macromolecular leakage $(n=5)$ & $0.7 \pm 0.08$ & $0.92 \pm 0.12$ \\
\hline Rolling leukocytes $(\mathrm{n}=5)(\mathrm{n} / \mathrm{mm} / \mathrm{s})$ & $8.3 \pm 1.9$ & $52.1 \pm 9.2$ \\
\hline Adherent leukocytes per $\mathrm{mm}^{2}(\mathrm{n}=5)$ & $24.1 \pm 13.2$ & $1137.5 \pm 612.2$ \\
\hline
\end{tabular}

venules. This technique allows for high-resolution images as a prerequisite for the precise quantification of the above in vivo parameters in various organs..$^{9,10,15,18,19}$

To date, only a few animal models are available for the investigation of the characteristics of $\mathrm{I} / \mathrm{R}$ of the urinary bladder. Some investigators have studied the role of ischemia in the urinary bladder in terms of smooth muscle function. Vanarsdalen et al. ${ }^{20}$ investigated the consequences of bladder ischemia in a rabbit model. After 1 hour of ischemia, they found a significant reduction in smooth muscle function of the bladder. Gill et al. ${ }^{21}$ observed a reduction in compliance and capacity of the urinary bladder after ischemic periods of different lengths. In an I/R study, Saito et al. ${ }^{22}$ described a decrease in the contractile response of the rat bladder to carbachol after several durations of ischemia $(0,30,60$, and 90 minutes) followed by reperfusion. The effect was time dependent; 60 minutes of ischemia caused considerable functional impairment that developed further during a subsequent reperfusion period of 30 minutes. In the same study, histologic examination revealed significant infiltration of leukocytes into the submucosal region and into the smooth muscle after 60 minutes of ischemia and 30 minutes of reperfusion. ${ }^{22}$ In addition to functional and structural alterations, an activation of both inducible and endothelial nitric oxide synthases was found with the same experimental design. ${ }^{23}$ The increase of intracellular $\mathrm{Ca}^{2+}$ after I/R may play an important role in these processes. ${ }^{24}$ Others reported that the mRNA levels of early response genes were elevated after an ischemic episode of the urinary bladder. ${ }^{25}$

Our study presents the first intravital microscopic analysis of I/R-induced inflammatory reactions in the microcirculation of the rat urinary bladder. I/R significantly impaired microvascular perfusion as evidenced by a loss of FCD, a lower blood flow velocity, and vasoconstriction in arterioles and venules that presumably was due to a preponderance of vasoconstrictors (eg, ET-1) dur- ing reperfusion. Concurrent with a decrease in diameter and blood flow velocity, the blood flow in the postcapillary venules was also reduced, indicating a relative hypoperfusion of the postischemic tissue. Leukocyte-endothelial cell interactions and endothelial permeability, both indexes of postischemic inflammation, were drastically enhanced. The histologic changes found after I/R of the urinary bladder were very similar to those observed in other I/R studies, as well as in other models of cystitis, in particular in models of interstitial cystitis.

Microvascular perfusion failure causing prolonged hypoxia, leukocyte infiltration, and interstitial edema as observed in the present study are factors underlying a potential subsequent injury of parenchymatous tissue.

In principle, the degree of I/R injury correlates positively with the duration of ischemia and reperfusion, respectively. In the present study, a combination of 60 minutes of ischemia and 30 minutes of reperfusion was chosen, because this regimen was previously found to cause structural and functional changes in the bladder effectively. ${ }^{22,23}$ Concern might exist that the I/R injury in our study could have been aggravated by atropine by binding to muscarinic receptors. However, in a previous study, we investigated the red blood cell velocity and FCD in the intact bladder wall under control conditions without atropine administration. ${ }^{26}$ The values found there were in close range or almost identical with the control values of the present study, hence atropine presumably had no effect on the results after I/R. Filling the bladder with a fixed volume facilitated IVM but also might have impaired perfusion. However, the applied volume of $0.5 \mathrm{~mL}$ did not exceed the physiologic capacity of the rat bladder. ${ }^{27}$ Moreover, the intravesical pressure was only about $9 \mathrm{~mm} \mathrm{Hg}$ in both groups and values of up to $70 \mathrm{~mm} \mathrm{Hg}$ previously did not result in impaired perfusion, at least under control conditions. ${ }^{26}$ 
IVM appears to be an excellent technique for the detailed investigation of the microcirculatory effects of drugs and compounds already used in research or in clinical practice. Therefore, this standardized, reliable model of an I/R-induced cystitis, in addition to answering the particular question addressed in the present study, represents a new methodologic approach for future investigations aimed at a better understanding of basic pathologic phenomena at the microcirculatory level.

\section{REFERENCES}

1. Alfieri $A B$, and Cubeddu LX: Nitric oxide and $\mathrm{NK}_{1}$ tachykinin receptors in cyclophosphamide-induced cystitis in rats. J Pharmacol Exp Ther 295: 824-829, 2000.

2. Dupont MC, Spitsbergen JM, Kim KB, et al: Histological and neurotrophic changes triggered by varying models of bladder inflammation. J Urol 166: 1111-1118, 2001.

3. Callsen-Cencic P, and Mense S: Expression of neuropeptides and nitric oxide synthase in neurones innervating the inflamed rat urinary bladder. J Auton Nerv Syst 65: 33-44, 1997.

4. Stein PC, Pham H, Ito T, et al: Bladder injury model induced in rats by exposure to protamine sulfate followed by bacterial endotoxin. J Urol 155: 1133-1138, 1996.

5. Zimmerman BJ, and Granger DN: Reperfusion injury. Surg Clin North Am 72: 65-83, 1992.

6. Von Dobschuetz E, Hoffmann T, and Messmer K: Inhibition of neutrophil proteinases by recombinant serpin lex032 reduces capillary no-reflow in ischemia/reperfusion-induced acute pancreatitis. J Pharmacol Exp Ther 290: 782-788, 1999.

7. Eppihimer MJ, and Granger DN: Ischemia/reperfusioninduced leukocyte-endothelial interactions in postcapillary venules. Shock 8: 16-25, 1997.

8. Szabo A, Boros M, Kaszaki J, et al: The role of mast cells in mucosal permeability changes during ischemia-reperfusion injury of the small intestine. Shock 8: 284-291, 1997.

9. Messmer K, and Krombach F: Microcirculation research in experimental surgery. Chirurg 69: 333-338, 1998.

10. Messmer K, Funk W, Endrich B, et al: The perspectives of new methods in microcirculation research. Prog Appl Microcirc 6: 77-90, 1984.

11. Zeintl H, Tompkins WR, Messmer K, et al: Static and dynamic microcirculatory video images analysis applied to clinical investigations. Prog Appl Microcirc 11: 1-10, 1986.
12. Zeintl H, Sack FU, Intaglietta M, et al: Computer assisted leukocyte adhesion measurement in intravital microscopy. Int J Microcirc Clin Exp 8: 293-302, 1989.

13. Grinberg-Funes D, Sheldon C, and Weiss M: The use of prostaglandin $\mathrm{F}_{2}$ for the prophylaxis of cyclophosphamideinduced cystitis in the rat. J Urol 44: 1500-1504, 1990.

14. Luber-Narod J, Austin-Ritchie T, Banner B, et al: Experimental autoimmune cystitis in the Lewis rat: a potential animal model for interstitial cystitis. Urol Res 24: 367-373, 1996.

15. Szabo A, Kaszaki J, Boros M, et al: Possible relationship between histamine and nitric oxide release in the postischemic flow response following mesenteric ischemia of different durations. Shock 7: 376-382, 1997.

16. Massberg S, and Messmer K: The nature of ischemia/ reperfusion injury. Transplant Proc 30: 4217-4223, 1998.

17. Granger DN, Rutili G, and McCord JM: Superoxide radicals in the feline intestinal ischemia. Gastroenterology 81: 22-29, 1981.

18. Menger MD, Marzi I, and Messmer K: In vivo fluorescence microscopy for quantitative analysis of the hepatic microcirculation in hamsters and rats. Eur Surg Res 23: 158$169,1991$.

19. Endrich B, Asaishi K, Götz A, et al: Technical report-a new chamber technique for microvascular studies in unanesthetized hamsters. Res Exp Med (Berl) 177: 125-134, 1980.

20. Vanarsdalen KN, Wein AJ, and Levin RM: The contractile and metabolic effects of acute ischemia on the rabbit urinary bladder. J Urol 130: 180-182, 1983.

21. Gill HS, Monson FC, Wein AJ, et al: The effects of shortterm in-vivo ischemia on the contractile function of the rabbit urinary bladder. J Urol 139: 1350-1354, 1988.

22. Saito M, Wada K, Kamisaki Y, et al: Effect of ischemiareperfusion on contractile function of rat urinary bladder: possible role of nitric oxide. Life Sci 62: PL149-PL156, 1998.

23. Saito M, and Miyagawa I: Direct detection of nitric oxide in rat urinary bladder during ischemia-reperfusion. J Urol 162: 1490-1495, 1999.

24. Zhao Y, Levin SS, Wein AJ, et al: Correlation of ischemia/reperfusion or partial outlet obstruction-induced spectrin proteolysis by calpain with contractile dysfunction in rabbit bladder. Urology 49: 293-300, 1997.

25. Chen MW, Buttyan R, and Levin RM: Genetic and cellular response to unilateral ischemia of the rabbit urinary bladder. J Urol 155: 732-737, 1996.

26. Bajory Z, Szabo A, Pajor L, et al: Intravital microscopic assessment of pressure induced microcirculatory changes after enterocystoplasty in rats. J Urol 165: 1279-1282, 2001.

27. Berggren T, and Uvelius B: Cystometrical evaluation of acute and chronic overdistension in the rat urinary bladder. Urol Res 26: 325-330, 1998. 